\title{
Design and Implementation of a Virtual Calculation Centre (VCC) for Engineering Students
}

\author{
doi:10.3991/ijoe.v6i1.1062
}

\author{
Alaeddine MOKRI \\ Abou Bekr Belkaid University, Tlemcen, Algeria
}

\begin{abstract}
The majority of academic institutions today provide their attendees with databases where courses and other materials could be uploaded, downloaded and checked by the faculty and students. Those materials are mostly PDF files, MS Word files, MS power point presentations or downloadable computer programs. This paper represents a new approach to providing engineering students with the data and the calculation programs. Engineering students need thermo-physical properties of substances, charts, diagrams, conversion factors and so forth. In addition, many students find it cumbersome downloading and installing superfluous computer programs which they do not need often in their studies. As an attempt to satisfy the academic community needs in the faculty of Engineering in Abou Bekr Belkaid University (Tlemcen, Algeria), we devised Web technologies and techniques to design an interactive virtual space wherein many engineering-related Web applications are accessible on-line. Students and professors can access on-line the properties of many substances, convert physical quantities from and into a variety of units, exploit computer programs on-line without installing them, generate tables and charts, and can use diagrams on-line by means of the mouse. The set of those applications is called a Virtual Calculation Center. This paper presents the different services that could be implemented in a Virtual Calculation Center, and describes the techniques and technologies used to build those applications.
\end{abstract}

Index Terms-Virtual Calculation Centre; on-line calculation; Web application; electronic chart; e-learning.

\section{INTRODUCTION:}

The acronym VCC ${ }^{1}$ which stands for "Virtual Calculation Centre" was coined and first introduced in the First Forum of Computer Engineering Students on June the $23^{\text {rd }}$ and $24^{\text {th }}, 2008$, in Bordj-Bou-Arreridj (Algeria) not as a technology in and of itself but a set of techniques and technologies that enable an on-line access to computer programs hosted on a server [1]. The techniques and the technologies used are all WWW-based. They have been exploited to develop scientific or engineering-related applications. In that year, and after discussing and polling a sample of developers, engineers and educators, the developer A.MOKRI launched a Website in the faculty of Engineering in Abou Bekr Belkaid University (Algeria) wherein many scripts and on-line calculation programs

1 The VCC stands for Virtual Calculation Center and it is accessible through the link: http://www.univ-tlemcen.dz/ dep-gm/CALCUL were accessible by the scientific community on the entire planet by means of any PC or laptop connected to Internet. The website is actually referenced by many research engines as "Centre de Calcul Virtuel". The image of the VCC $^{1}$ has evolved from a concept to a tool for integrating e-learning applications and to a wide virtual space where scientists and engineers could access to calculation programs, charts, tables, on-line units converters and other applications. [2]

The VCC was purposely designed to assist students and faculty alike in their studies and research by providing them by online accessible services.

Literature review on the previous projects done to use WWW-based technologies to assist engineering students and engineers has shown many interesting initiatives and much development along the last 15 years.

In 1994, a Website was created by the Northern Illinois University for chemistry students. Many services were provided: email address directory, chemistry software database, conference listings, electronic conferencing and other services were prospected. The motive behind this work was to use the Internet for academic purposes, and get an insight into the potential benefits [3].

Some years later, a WWW-based control system of a synchrotron radiation facility was designed. The purpose was to devise a way to keep the practitioners far from the dangerous zones near the facility [4]. Since then, many remotely controlled systems have been designed for the same purpose.

A different approach to using software was suggested for calculation of energy and cost demands for heating, cooling and hot water in homes in the USA. The approach was to design a package consisting of many routines and scripts coded in four languages: HTML, Perl, C and Fortran and accessible online [5]. This approach expanded widely the accessibility because the package was available online and people were able to access to it and use it. This package was designed for estimating energy use in 900 US cities.

In the last years, as an attempt to extend access to engineering for the disabled, many universities have created their own or shared remote laboratories. By using WWW-based technologies, students with disabilities have been able to run real-world experiments remotely and safely [6-8].

Much development in the WWW-based technologies has led to the advent of many sophisticated tools. In the Web, one can find many Websites that offer services for 
engineering students, professors and engineers. Among them, one has to mention the MIT OpenCourseWare [16], which consists in a Website from which users can access to many courses in different formats (video recordings, pdf and MS Word files) but it does not provide any online calculation services.

The other source is the Virtual Laboratory [17]. It offers access to many Java applets to simulate physical phenomena. Users can access online to the Website and run the applets to simulate a given physical phenomenon under different conditions.

The Martindale's Calculators On-line Center [18] is a Website that offers the links and the description of the different Websites dedicated to on-line calculations. The problem is that even though those kinds of Websites are numerous, they offer the same kind of services with no noticeable differences beside the graphical design.

In addition to the sources stated earlier, one can find many other interesting sources on the net. In this work, the focus is only the sources that offer online calculation services.

As a glance at those sources that offer on-line calculation services, the developers generally use one technique for implementing the computer programs. Some developers use only Java applets to make simple graphical simulations of physical phenomena, and other developers use only CGI scripts to develop programs that enable the user to draw curves or make mathematical calculations. In addition, the Webmasters offer services to a restricted community of users, such as: physicists, mathematicians and so on.

Besides, those on-line accessible programs perform the same calculations that conventional software perform. Technically speaking, the on-line applications should use the available Web technologies to add a value to the conventional locally executable computer programs.

Users of engineering-related software should always look for extra-files to update their products.

Below is our contribution and the assets of the VCC that position it as an efficient tool to implement engineering-related Web applications:

- The VCC is not restricted to a specific community, but all the scientific community can exploit it because it encompasses a variety of programs and services.

- Some calculations require that the user knows the real circumstances in which a given system operates such as: altitude, latitude, time. So if the user does not know those parameters, erroneous results might be achieved. However, in the VCC, the programs are designed to interact with the user according to his IP address, that is to say, the geographical location and time.

- Even more importantly, unlike conventional on-line calculation websites, the VCC enables the user to develop his own applications and programs to exploit them later.

- The user always has access to the updated version of the programs, without downloading any updates.

The data is transferred using the Transmission Control Protocol/Internet Protocol (TCP/IP). Information on the Internet is located on the server. Locating and retrieving the data are the tasks of the browser. Internet Explorer 6.0 is considered to be the browser because it is installed in all the computers in Tlemcen University.

In this article, the format as well as the many services that are available in the Virtual Calculation Center are described, as well as, there benefits. After that, the author goes to list the different techniques and technologies used to create and implement the different applications, such as the programming languages and the Web techniques.

\section{THE FORMAT OF THE WEBSITE:}

Because all the courses are delivered in French in Tlemcen University, the Webmaster has used the French language in the VCC, so the students use the VCC comfortably. In addition to this, most the Websites that offer online calculation services are in English, so the Webmaster would like to offer those services to people who feel more comfortable with the French language.

To put it clearly, the Webmaster must consider the linguistic background of the potential users.

The Website does not contain many graphics, that is why the Web pages do not take a lot of time to load. The Webmaster tried to avoid the use of graphics because sometimes the bandwidth is very low, and hence, it takes a long time to access to the Webpage.

But if the bandwidth is not an issue, it is better to include graphics to make the Webpage (i.e. the graphical user interface) more interactive.

The font and the size of the items were adjusted to be easily recognized by the users. The VCC may be accessed by means of a small device (i.e. mobile phone, personal digital assistant), so the items must be readable from this kind of devices.

The main Webpage should include all the links to the different Web pages, so the user can access to all the available services. Similarly, all the Web pages should include a link to the related Web pages that offer similar services and to the main Webpage as well.

The main Webpage consists of many frames. Each frame contains the links to the Web pages that offer similar features. For example, the links to all the pages that offer thermo-physical properties should be gathered in one frame.

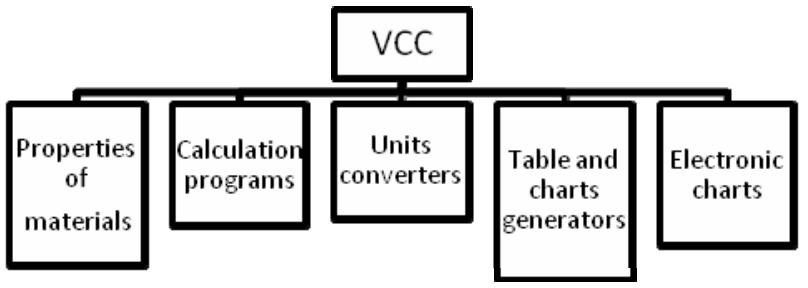

Figure 1. The different applications in the VCC.

\section{Designing the Virtual Calculation CEnTRE:}

As previously mentioned, to design a $\mathrm{VCC}^{1}$ is to make use of all the available techniques and technologies in order to make calculation computer programs accessible via a network. In addition, in some specific cases, programs should interact with the user according to the surrounding environment. The term environment, in this context, has connotations of: time, geographic location, browser, installed programs. 
The components of the Virtual Calculation Centre are listed below and the list is not limited, because the provided services are originally some ideas that the Webmaster sees very helpful for students. For the case being, the targeted community is the students of an engineering faculty. For another community, the services that should be provided may differ. The figure below shows the applications in the VCC.

\section{A. Properties of Substances:}

Working on a scientific project, teaching, solving a technical problem inherently requires the determination of the thermophysical properties of substances (i.e. materials, metals, alloys, liquids, gases and so forth).

In the VCC, more than thirty (30) thermo-physical properties of many solids, gases and liquids are accessible on-line.

First, it was necessary to make a collection of properties of the most frequently encountered materials, gases and liquids by engineering students. At that stage, we relied on some books devoted only to materials properties, as well as some appendices in some books. After that, a process of arranging the data and investigating their accuracy was gone through. Later, we integrated the properties in JavaScript scripts and an HTML Webpage was designed to host the forms whereby users can select a material and after clicking on the button, the properties appear immediately in the screen (Figure 2).

In the VCC, chemical and physical properties of thousands of substances are accessible on-line whenever and wherever which are not available elsewhere. One important thing is the design of the Webpage, so the developer has to arrange the properties in such a manner that the user can easily find them. For instance, the pages corresponding to the thermal properties were all arranged together, and the ones related to chemical properties were also arranged together in the main Webpage of the VCC. In addition, those properties are always to be updated.

\section{A. Electronic Charts:}

The electronic chart is a new innovative solution devised by the Webmaster to enable an accurate on-line use of the charts. The application consists on a Webpage where a chart is displayed and the user can use the chart on-line by means of the mouse. As a first evaluation of the idea, a psychometric chart was used (see figure 3 ). The user, by moving the mouse cursor over the chart, can instantaneously determine the data of any point on the chart after clicking on a given point or by simply moving the cursor over it. For the sake of accuracy, the source code may need to be altered to adapt the real dimensions of the chart to the dimensions of the screen.

\section{B. Tables and Charts Generators:}

In the engineering realm, tables and charts where properties of a given substance are enumerated are always required. Usually, charts and tables include properties of a given substance under different conditions, as for example the temperature and density of air for different altitudes. Those tables may be found in appendices of some books, and users usually prefer a printed copy of those documents to work with in classrooms or laboratories. Sometimes, those tables do not cover the range of properties in which the Engineer or the student works. In addition, if the gap between subsequent values is large, so the user has to

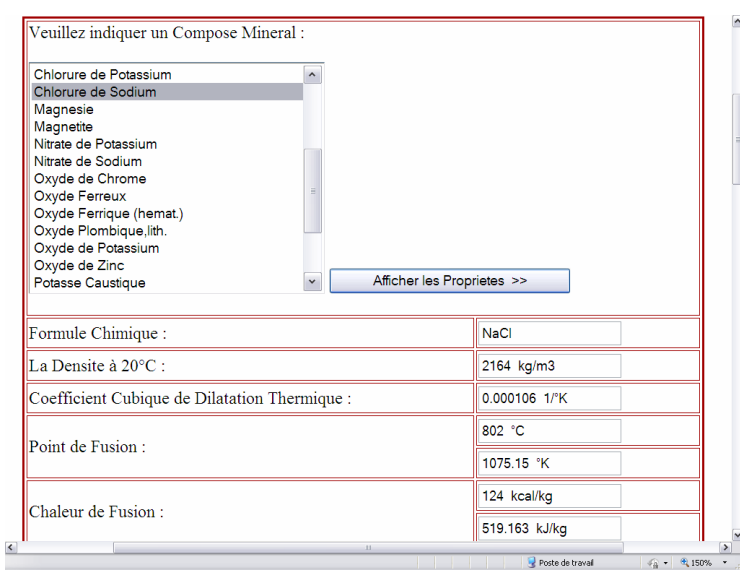

Figure 2. Thermo-physical properties of a substance displayed in the VCC Webpage.

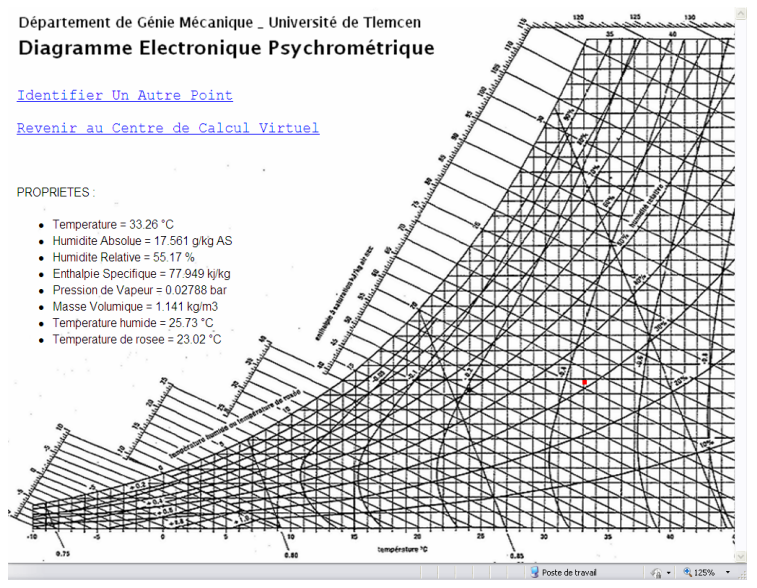

Figure 3. An electronic chart for calculating air properties.

make some correlations which affect the accuracy of the data.

However, in the VCC, there are many programs that generate tables and charts according to the user's needs (range, gap between subsequent properties).

First of all, we had to poll students, professors and engineers about the charts and the tables usually needed in their projects. Once done, those tables were investigated and by using appropriate interpolation algorithms, mathematical formulas and algorithms that describe all the tabulated values were achieved. For many properties, those correlation formulas were just brought from some materials. By means of the form and JavaScript scripts, the user can type the first and the last values that limit the interval within he/she works and then type in the step between the successive values and after selecting the appropriate substance and clicking on the button (see figure 4), a table appears immediately including the properties of the selected substance (see figure 5). Eventually, the user can print the table to work with it on its own pace.

In the VCC, three table generators are available so far, but many of them are prospected.

\section{Tables of Properties (the periodic table):}

In literature, properties of many materials are tabulated and arranged in a specific way. One example is the periodic table where elements are tabulated and arranged in such a way that their position in the table reflects their 


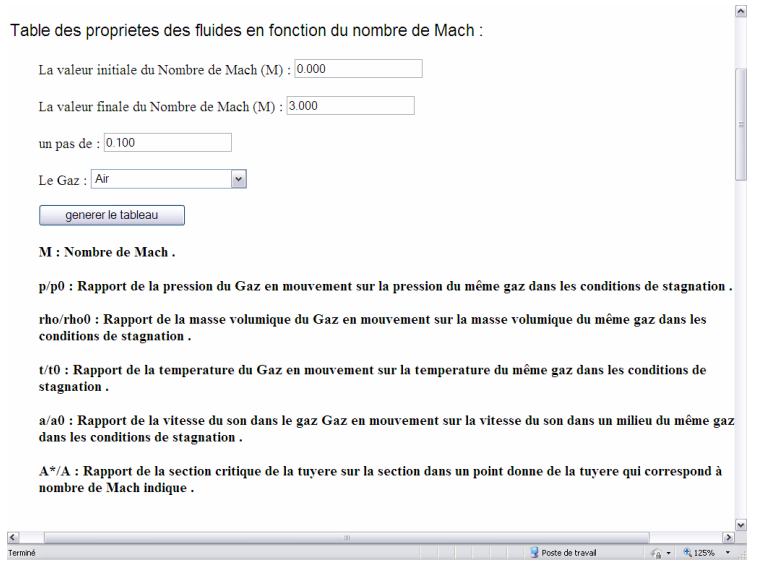

Figure 4. A form to generate properties of a substance in a table.

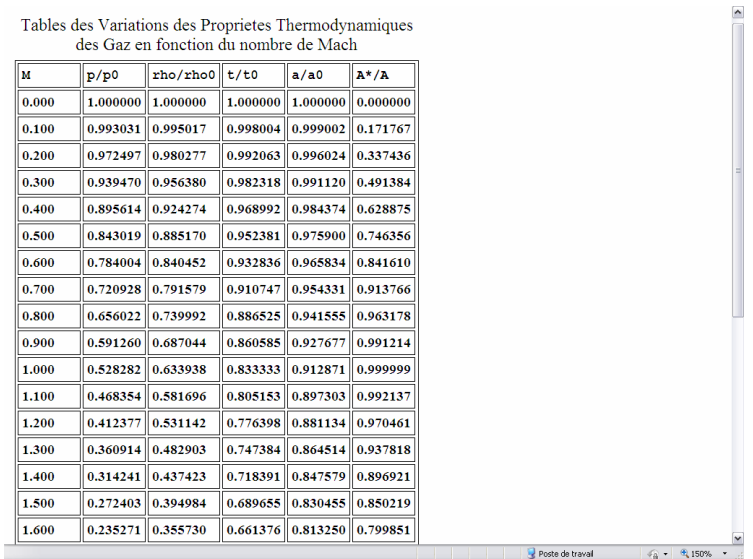

Figure 5. Properties of a substance tabulated in the generated table.

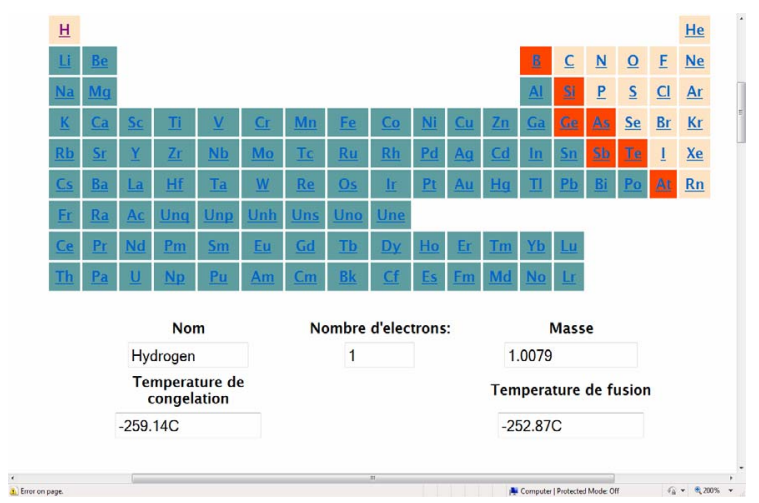

Figure 6. Properties of a substance tabulated in the generated table.

properties (number of protons, number of electrons, electro-negativity, number of shells and sub-shells and so on). But to determine some of those properties, one may require a deep understanding of materials properties and their physics [9].

In the VCC, a periodic table was created. So the user has to click on the box that corresponds to the element, and then, its properties are displayed in the form. Even though the user may be unable ? the properties of the elements according to their positions in the table, by using the form, that task is very easy and practical.

\section{Calculation Program:}

The commonplace calculation programs executable locally in the PC could be programmed again as scripts or programs that could be accessible on-line. When it comes to calculation programs, the task is to be let for specialists to come out with accurate and efficient solutions. The programs could be categorized into two classes: complex and simple ones. For simple programs, one has to determine whether it is better to use Perl, VB or JavaScript scripts. But for complex programs such as the ones that generate graphics and curves or programs that involve complex algorithms, CGI Scripts are usually suitable. In order to write CGI scripts, C, C++ and FORTRAN are mainly used as languages of programming. The means of interaction is always a form where the user has to indicate the appropriate values and after he/she clicks on the button, the results will be displayed in the same form (figure 7). So far, six calculation programs are accessible in the VCC.

In this kind of program, the developer, and for specific cases, has to provide the user by applications that take in consideration the real-time circumstances. For instance, to use a program for calculating a solar settlement, with CGI scripts written in FORTRAN, the user does not have to to indicate the actual time and the actual geographic location because those values are automatically determined by the program.

With this same technique, we have created more than 45 on-line unit converters, most of them offer 64 possibility of conversion (see figure 8), what we refer to as a large (the largest?) collection of on-line converters on the Internet. Here, one should admit that there is no tool besides the VCC that can offer such a good service to users.

\section{E. On-line calculators:}

Students need calculators in their work. They mainly use normal scientific calculators or the one installed in their computers. In the VCC, a scientific calculator was created by using JavaScript scripts, and an HTML form. It has the same features as the traditional calculators.(see figure 9)

\section{TEChNiques AND TEChNOLOGIES:}

Web technologies are evolving and improving dramatically, and it is one of the telling reasons to exploit them for developing engineering-related applications.

To build a Virtual Calculation Centre, one has to intentionally choose the most appropriate technique because for each application there is one technique which is easy to implement and exploit.

In this section, we are going to list the techniques and the programming languages that we usually use to develop on-line applications.

\section{A. VB, JavaScript: [10, 11, 12]}

The way scripts work is quite simple. First of all, the developer has to design an interactive form by means of HTML instructions, in which the user can inject the data. The form consists of the means that enable the user to provide the program with data such as text zones and selection lists. The script is either hosted in the server in a separate file and a link to the script is inserted in the HTML code, or it is integrated as a series of instruction in the HTML code. In the latter case, the script should be inserted in the part called "head" in the HTML code, so the script will be loaded first which prevents from errors of execution. 


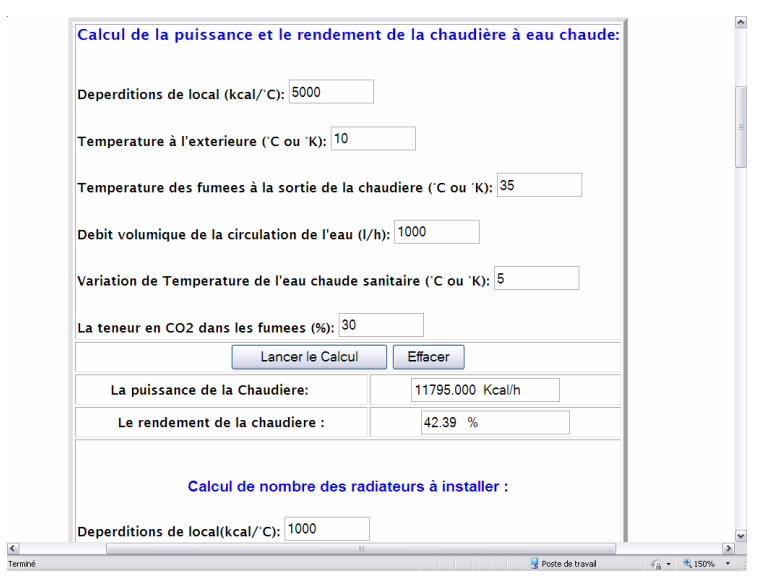

Figure 7. A form for calculating and assessing the performance of a settlement.

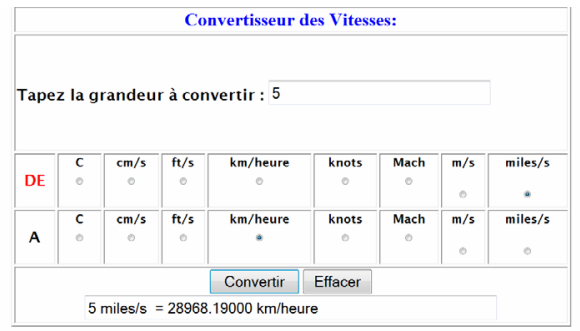

Figure 8. On line units converter.
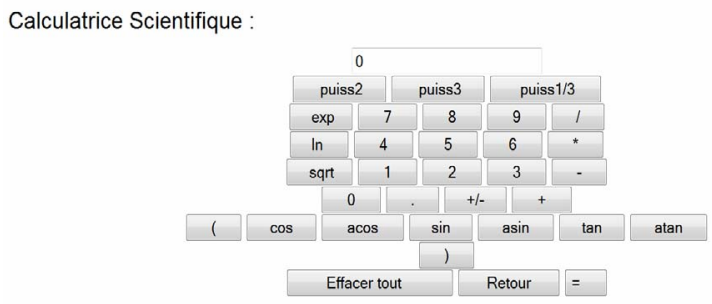

NOTE: Les angles doivent être exprimees en radians.

Figure 9. A scientific calculator

JavaScript is more suitable than VB scripts in the case of programming mathematical formulas.

We use JavaScript to implement simple algorithms that does not contain complex and long instructions. With JavaScript, we can manage to write a script that recuperates automatically information about the environment of the client. [13]

\section{B. Perl:}

Working with Perl is as working with JavaScript and VBScript; in addition, it is practical for creating applications that involve characters as variables. We usually use Perl for medical purposes for deciphering DNA or RNA chains.

Perl can be used for applications that involve generating curves because of the multiple graphical libraries that can be worked with in Perl scripts.

\section{CGI (Common Gateway Interface):}

The CGI scripts are vital for developing on-line applications. They are used for developing complex applications. The CGI script is a program written usually in FORTRAN, C, C++ and hosted in the server in the repertory called (cgi-bin), and by means of a form, the user inserts the data that will be sent to the program in the repertory, and then the program returns the result either to the form, to another webpage, or the mailbox of the user [14].

The second reason, for which we use CGI scripts, is that we can determine the geographic location of the user according to the IP address. In addition, the CGI script enables us to develop interactive graphical applications such as drawing curves and diagrams, generating images. [15]

\section{Applets JAVA:}

Applets JAVA are useful for creating some applications of simulating physical phenomena. The animations are interactive because the user can, by means of the mouse, interact with the graphic and observe the evolution of systems and mechanisms. However, the development and the implementation of JAVA applets are comparatively hard tasks and even the browsers may find problems to load the animations.

\section{CONCLUSIONS:}

After designing and implementing the VCC, we have succeeded in achieving our goal which is programming, gathering, arranging and making accessible new sources (data and tools) that engineering students and professors need in their projects.

Instead of looking for the data in different sources (books, web, charts and so on), the VCC enables the user to find all that he/she needs, in one location, whenever and wherever.

What is more, the most important benefit we have drawn from this experience is the fact that we are promoting a new concept that consists of programming, gathering, arranging and making accessible all what students and engineers need. This concept is to be worked in e-learning and Web service applications.

Finally, the VCC is a new concept in the commercialization of programmed calculation tools (software) and data (e.g. materials properties).

\section{NOTE:}

The VCC website mentioned throughout this study is accessible via the link below:

\section{http://www.univ-tlemcen.dz/ dep-gm/CALCUL}

\section{ACKNOWLEDGMENT}

The author would like to acknowledge "Le centre de tele-enseignement" in Tlemcen University for hosting the VCC.

\section{REFERENCES:}

[1] A. Mokri, "Réalisation d'un centre de calcul virtuel pour le calcul en ligne,” Recueil des Communications du 1er Forum National des Etudiants en Informatique 23-24 Juin 2008, Centre Universitaire de Bordj Bou Arreridj. Accessible here: http://publications.univ-tlemcen.dz/spip.php?article485 
[2] A. Mokri, "Case-Study: Design and Implementation of a Virtual Calculation Centre (VCC) in Abou Bekr Belkaid University.” Proceedings of the sixth international conference on remote engineering and virtual instrumentation, June 22-25, 2009, Bridgeport University, USA.

[3] S. M. Bachrach, "Chemistry on the Internet: The Northern Illinois University Chemistry WWW/Gopher Site," trends in analytical chemistry, 14 (5) 1995, pp 182-185.

[4] F. Bille, R. Pugliese, "Using WWW technology in a control system," Nuclear instruments and methods in physics research A 389 (1997), pp 114-116. (doi:10.1016/S0168-9002(97)00058-2)

[5] T. Forowicz, "Modeling of energy demands for residential buildings with HTML interface," Automation in construction 8 (1999) 481-487. (doi:10.1016/S0926-5805(98)00095-8)

[6] C. Colwell, E. Scanlon, M. Cooper, "Using remote laboratories to extend access to science and engineering," Computers and education 38 (2002) 65-76. (doi:10.1016/S0360-1315(01)00077X)

[7] P. Arpaia, A. Baccigalupi, F. Cennamo, P. Daponte, " A remote measurement laboratory for educational experiments," Measurements Vol. 21, No. 4, 1997, pp 157-169. (doi:10.1016/ $\underline{\text { S0263-2241(97)00062-6) }}$

[8] K. Yeung, J. Huang, "Development of a remote-access laboratory: a dc motor control experiment," Computers in industry 52 (2003) 305-311. (doi:10.1016/S0166-3615(03)00133-7)

[9] William D. Callister, "Fundamentals of Materials Science and Engineering,” John Wiley \& Sons, Inc. ,2001.
[10] David R. Brooks, "An Introduction to HTML and JavaScript for Scientists and Engineers,” Springer, 2006.

[11] Steven Holzner, “Inside JavaScript,” New Riders Publishing, 2002.

[12] Scott Duffy, "How to do everything with JavaScript," McGrawHill, 2003.

[13] Chuck Easttom, "Learn JavaScript" Wordware Publishing, Inc, 2002.

[14] Eric Eugene, “CGI Developer's Guide,” Sams.net Publishing, July-1996.

[15] Robert McDaniel Macmillan, “CGI Manual of Style” Computer Publishing USA, 1996.

[16] http://ocw.mit.edu/OcwWeb/web/home/home/index.htm

[17] http://e.m.c.2.free.fr/applets.htm

[18] http://www.martindalecenter.com/Calculators.htm]

\section{AUTHORS}

A. Mokri was with the Department of Mechanical Engineering, Abou Bekr Belkaid University, Chetouane P.O. Box 230, Tlemcen, Algeria. He is now with Masdar Institute of Science and Technology P.O. Box: 54224, Abu Dhabi, UAE (alaeddinemokri@gmail.com).

Manuscript received 18 September 2009. Published as submitted by the author January 20, 2010. 